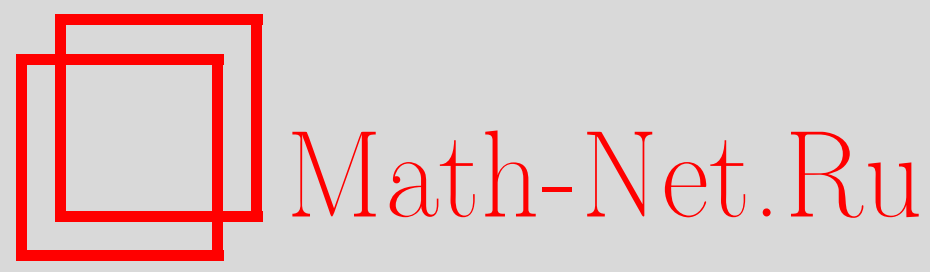

С. С. Марченков, О суперпозициях непрерывных функций, заданных на бэровском пространстве, Матем. заметки, 1999, том 66, выпуск 5, 696-705

DOI: https://doi.org/10.4213/mzm1214

Использование Общероссийского математического портала Math-Net.Ru подразумевает, что вы прочитали и согласны с пользовательским соглашением http://www . mathnet.ru/rus/agreement

Параметры загрузки:

IP: 3.80 .253 .173

26 апреля 2023 г., 06:31:50

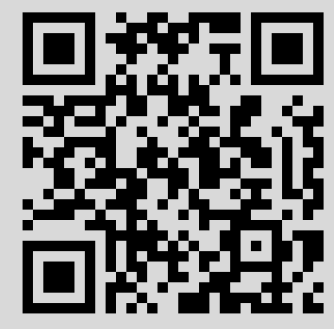




\title{
О СУПЕРПОЗИЦИЯХ НЕПРЕРЫВНЫХ ФУНКЦИЙ, ЗАДАННЫХ НА БЭРОВСКОМ ПРОСТРАНСТВЕ
}

\author{
С. С. Марченков
}

\begin{abstract}
Для непрерывных функций, заданных на бэровском пространстве, рассматривается следующая задача: при каких условиях функции нескольких переменных можно реализовать суперпозициями функций меньшего числа переменных. С помощью линейных функций вида $(1+\alpha) t$ определяется граница для модуля непрерывности, отделяющая положительное решение задачи от отрицательного. В случае отрицательного решения предлагается конструктивный способ построения $(n+1)$-местных непрерывных функций с модулем непрерывности $\varphi(t)$, которые невозможно представить в виде суперпозиции $n$-местных непрерывных функций с тем же модулем непрерывности $\varphi(t)$.

Библиография: 12 названий.
\end{abstract}

В различных разделах математики часто возникает следующая задача, связанная с суперпозициями функций. Пусть $M$ - некоторый класс функций, $M^{(n)}$ - множество всех $n$-местных функций из $M,\left[M^{(n)}\right]$ - множество всех функций, реализуемых суперпозициями функций из $M^{(n)}$. Верно ли, что при любом $n, n \geqslant 2$, вьполняется включение $M^{(n+1)} \subset\left[M^{(n)}\right]$ ?

Если $E$ - непустое множество и $M_{E}-$ класс всех функций, определенных на $E$, то при любом $n, n \geqslant 2$, имеет место включение $M_{E}^{(n+1)} \subset\left[M_{E}^{(2)}\right]$ (для конечных множеств $E$ соответствуюшие утверждения являются следствиями важных теорем о разложении функций многозначной логики [1]). Однако для многих содержательно интересных классов функций $M$ вопрос о соотношении множеств $M^{(n+1)}$ и $\left[M^{(n)}\right]$ оказьвается достаточно сложньм. Так, например, обстоит дело с различными классами непрерывных функций. Если на непрерывные функции не налагать никаких дополнительных ограничений, то всякую многоместную непрерьвную функцию можно реализовать в виде суперпозиции подходящих одно- и двуместных непрерывных функций (решение этой проблемы см. в [2]-[4]). Напротив, если функции из $M$ удовлетворяют некоторьп условиям гладкости, то соотношение $M^{(n+1)} \subset\left[M^{(n)}\right]$ не выполняется ни при каком $n, n \geqslant 2,[5]-[11]$.

В работе [10] предложен некоторый метод анализа суперпозиций непрерьвных функций, использующий булевы функции и сложность реализации булевых функций схемами из функциональных элементов. Как видно из [10], в наиболее прозрачной форме метод применим к так назьваемым детерминированным функциям - частному случаю непрерьвных функций, заданных на бэровском пространстве. В настоящей статье мы рассматриваем эти функции в общем случае и решаем вопрос о выразимости функций многих переменных в виде суперпозиции функций меньшего числа переменных. В основных

Работа выполнена при финансовой поддержке Российского фонда фундаментальных исследований, грант № 97-01-00989. 
результатах (теоремы 1-3) с помощью модуля непрерывности определяется граница, которая отделяет положительное решение вопроса от отрицательного. Как и в работе [10], в качестве основного аппарата исследований используются булевы функции, а также приближения непрерьвных функций булевскими операторами.

Дадим необходимые определения. Пусть $I=\{0,1\}^{\infty}$ - декартово произведение счетного числа множеств $\{0,1\}$. Элементы $a, b$ множества $I$ будем записьвать в виде $a=$ $a(1) a(2) \ldots, b=b(1) b(2) \ldots$, где $a(t), b(t) \in\{0,1\}$ при $t \geqslant 1$. На множестве $I$ зададим метрику $\rho$ бэровского пространства: $\rho(a, b)=1 / t$, если $a \neq b$ и $t$ есть такое наименьшее число, что $a(t) \neq b(t)$. Метрику $\rho$ перенесем на декартовы степени множества $I$ : если $\mathbf{a}, \mathbf{b} \in I^{n}, \mathbf{a}=\left(a_{1}, \ldots, a_{n}\right), \mathbf{b}=\left(b_{1}, \ldots, b_{n}\right)$, то $\rho(\mathbf{a}, \mathbf{b})=\max _{1 \leqslant i \leqslant n} \rho\left(a_{i}, b_{i}\right)$.

Будем рассматривать функции $f\left(x_{1}, \ldots, x_{n}\right)$, отображающие $I^{n}$ в $I$. По техническим причинам для оперирования с функцией $f\left(x_{1}, \ldots, x_{n}\right)$ удобно ввести еще одну переменную $y: y=f\left(x_{1}, \ldots, x_{n}\right)$. В этих обозначениях при любом $t, t \geqslant 1, y(t)$ является функцией, вообще говоря, бесконечного числа булевских переменных $x_{1}(1), \ldots, x_{n}(1)$, $x_{1}(2), \ldots, x_{n}(2), \ldots$ Нетрудно, однако, видеть, что для определенной вьше метрики $\rho$ функция $f\left(x_{1}, \ldots, x_{n}\right.$ ) (равномерно) непрерьвна в том и только том случае, когда существует такая функция $\varphi(t)$ натурального аргумента $t$, принимающая натуральные значения, что при любом $t, t \geqslant 1, y(t)$ определяется только значениями $x_{1}(1), \ldots, x_{n}(1), \ldots$, $x_{1}(\varphi(t)), \ldots, x_{n}(\varphi(t))$. Иными словами, для любого $t$ должна существовать такая булева функция $G_{t}$ от $n \varphi(t)$ переменных, что

$$
y(t)=G_{t}\left(x_{1}(1), \ldots, x_{n}(1), \ldots, x_{1}(\varphi(t)), \ldots, x_{n}(\varphi(t))\right) .
$$

Функцию $\varphi(t)$ с указанными свойствами будем называть модулем непрерывности функции $f$. Если $\varphi(t)$ - модуль непрерывности функции $f$ и $\psi(t) \geqslant \varphi(t)$, то функцию $\psi(t)$ также можно считать модулем непрерьвности функции $f$. В связи с этим и в целях упрощения дальнейших построений модули непрерьвности будем предполагать монотонно неубываюшими функциями.

Обозначим через $\mathbf{C}$ множество всех непрерывных на $I$ функций. Пусть $\mathbf{C}_{\varphi}-$ подмножество $\mathbf{C}$, состоящее из функций с модулем непрерьвности $\varphi, \mathbf{C}^{(n)}-$ множество всех $n$-местных функций из $\mathbf{C}, \mathbf{C}_{\varphi}^{(n)}=\mathbf{C}_{\varphi} \cap \mathbf{C}^{(n)}$.

Пусть $M \subseteq \mathbf{C}$. Стандартным образом [12] определяется понятие формуль над $M$. Любая функция из $M$ является формулой над $M$. Если $f\left(x_{1}, \ldots, x_{n}\right)$ - функция из $M$, а $F_{1}, \ldots, F_{n}$ - либо формулы над $M$, либо символы переменных (не обязательно различные), то выражение $f\left(F_{1}, \ldots, F_{n}\right)$ также является формулой над $M$.

Каждая формула над $M$ очевидным образом задает некоторую функцию (вообще говоря, не принадлежащую $M)$. Множество всех функций, которые определяются всеми формулами над $M$, обозначается через $[M]$ и называется множеством функций, реализуемых суперпозициями функций из $M$, или, короче, - суперпозиииями функиий из $M$.

Для любого натурального $k$ пусть $\varphi^{(k)}(t)$ обозначает $k$-кратную суперпозицию функции $\varphi: \varphi^{(k)}(t)=\varphi(\varphi(\ldots \varphi(t) \ldots))$, где правая часть равенства содержит $k$ символов $\varphi$.

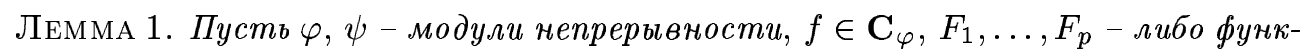
иии из $\mathbf{C}_{\psi}$, либо символы переменных и

$$
g\left(x_{1}, \ldots, x_{m}\right)=f\left(F_{1}, \ldots, F_{p}\right) .
$$

Тогда $g \in \mathbf{C}_{\tau}$, где $\tau(t)=\max (\varphi(t), \psi(\varphi(t)))$. 
ДокАЗАТЕЛЬСТво. В самом деле, пусть

$$
y=g\left(x_{1}, \ldots, x_{m}\right), \quad y_{1}=F_{1}, \ldots, \quad y_{p}=F_{p}
$$

Тогда согласно включению $f \in \mathbf{C}_{\varphi}$ значения $y(1), \ldots, y(t)$ полностью определяются значениями $y_{1}(1), \ldots, y_{p}(1), \ldots, y_{1}(\varphi(t)), \ldots, y_{p}(\varphi(t))$. Если $F_{i}(1 \leqslant i \leqslant p)$ есть функция из $\mathbf{C}_{\psi}, F_{i}=f_{i}\left(x_{1}^{i}, \ldots, x_{m_{i}}^{i}\right)$, где $\left\{x_{1}^{i}, \ldots, x_{m_{i}}^{i}\right\} \subseteq\left\{x_{1}, \ldots, x_{m}\right\}$, то в свою очередь $y_{i}(1), \ldots, y_{i}(\varphi(t))$ полностью определяются значениями

$$
x_{1}^{i}(1), \ldots, x_{m_{i}}^{i}(1), \ldots, x_{1}^{i}(\psi(\varphi(t))), \ldots, x_{m_{i}}^{i}(\psi(\varphi(t))) .
$$

Если же $F_{i}$ есть переменная $x_{j}$, то последовательность $y_{i}(1), \ldots, y_{i}(\varphi(t))$ совпадает с последовательностью $x_{j}(1), \ldots, x_{j}(\varphi(t))$. Отсюда вытекает утверждение леммы.

СЛЕДСТВИЕ 1. Имеем $[\mathbf{C}]=\mathbf{C}$.

СЛЕДСТВИЕ 2. Если $\varphi(t) \leqslant t, m o\left[\mathbf{C}_{\varphi}\right]=\mathbf{C}_{\varphi}$.

ДокАЗАТЕльСтво. Включение $\mathbf{C}_{\varphi} \subseteq\left[\mathbf{C}_{\varphi}\right]$ вьполняется по определению множества $\left[\mathbf{C}_{\varphi}\right]$. Для доказательства обратного включения достаточно рассмотреть элементарную суперпозицию вида $(2)$. Так как $\varphi(t) \leqslant t$ и функция $\varphi$ монотонно не убывает, то $\varphi^{(2)}(t) \leqslant \varphi(t)$. Значит, функция $g$ из $(2)$ принадлежит классу $\mathbf{C}_{\varphi}$.

Пусть $l$-натуральное число. Обозначим через $\mathbf{C}_{\varphi}^{[l]}$ множество всех функций, которые можно реализовать формулами над $\mathbf{C}_{\varphi}$, состоящими из $l$ символов функций.

СлЕДСТВИЕ 3. Eсли $\varphi(t) \geqslant t, m o \mathbf{C}_{\varphi}^{[l]} \subset \mathbf{C}_{\varphi^{(l)}}$.

ДокАЗАТЕЛЬСТво. Проведем индукцию по $l$. При $l=1$ утверждение вьполняется очевидньм образом. Пусть $l \geqslant 2$ и утверждение верно для меньших значений $l$. Если $g \in \mathbf{C}_{\varphi}^{[l]}$, то функция $g$ представима в виде $(2)$, где $f \in \mathbf{C}_{\varphi}$, a $F_{1}, \ldots, F_{p}$ - либо символы переменных, либо функции из класса $\mathbf{C}_{\varphi}^{[1]} \cup \cdots \cup \mathbf{C}_{\varphi}^{[l-1]}$. Если $F_{i}-$ функция из класса $\mathbf{C}_{\varphi}^{[k]}$, где $1 \leqslant k<l$, то в силу индуктивного предположения $F_{i} \in \mathbf{C}_{\varphi^{(k)}}$. Из неравенства $\varphi(t) \geqslant t$ и монотонности функции $\varphi$ следует, что $\mathbf{C}_{\varphi^{(k)}} \subseteq \mathbf{C}_{\varphi^{(l-1)}}$. Остается применить лемму 1 и еще раз воспользоваться неравенством $\varphi(t) \geqslant t$ и монотонностью функции $\varphi$.

Хорошо известно, что любые два множества $I^{m}, I^{n}(m, n \geqslant 1)$ гомеоморфны. Взаимно-однозначное и непрерьвное в обе стороны соответствие между $I^{2}$ и $I$ можно осуществить, например, используя следующую функиию Пеано $y=P\left(x_{1}, x_{2}\right)$ : если $x_{1}=$ $x_{1}(1) x_{1}(2) \ldots, x_{2}=x_{2}(1) x_{2}(2) \ldots$, то при любом $t, t \geqslant 1, y(2 t-1)=x_{1}(t), y(2 t)=$ $x_{2}(t)$. Пусть $y_{1}=p_{1}(x), y_{2}=p_{2}(x)$ - такие функции, что при любом $t, t \geqslant 1, y_{1}(t)=$ $x(2 t-1), y_{2}(t)=x(2 t)$. Имеем, очевидно, тождества:

$$
p_{1}\left(P\left(x_{1}, x_{2}\right)\right)=x_{1}, \quad p_{2}\left(P\left(x_{1}, x_{2}\right)\right)=x_{2}, \quad P\left(p_{1}(x), p_{2}(x)\right)=x .
$$

Из определений функций $P, p_{1}, p_{2}$ следует, что $P \in \mathbf{C}_{\varphi}$, где $\varphi(t)=\lceil t / 2\rceil$, и $p_{1}, p_{2} \in \mathbf{C}_{2 t}$. Таким образом, $\left\{P, p_{1}, p_{2}\right\} \subset \mathbf{C}_{2 t}$.

На основе функции $P$ стандартным образом устанавливается гомеоморфизм множеств $I^{n}$ и $I, n \geqslant 3$. Именно, положим $Q_{2}\left(x_{1}, x_{2}\right)=P\left(x_{1}, x_{2}\right)$ и при $n \geqslant 2$

$$
Q_{n+1}\left(x_{1}, \ldots, x_{n+1}\right)=Q_{2}\left(Q_{n}\left(x_{1}, \ldots, x_{n}\right), x_{n+1}\right) .
$$


Если определить далее $q_{1}^{2}(x)=p_{1}(x), q_{2}^{2}(x)=p_{2}(x)$ и

$$
q_{1}^{n+1}(x)=q_{1}^{n}\left(q_{1}^{2}(x)\right), \ldots, \quad q_{n}^{n+1}(x)=q_{n}^{n}\left(q_{1}^{2}(x)\right), \quad q_{n+1}^{n+1}=q_{2}^{2}(x),
$$

то при любом $n, n \geqslant 2$, будем иметь тождества

$$
\begin{gathered}
q_{1}^{n}\left(Q_{n}\left(x_{1}, \ldots, x_{n}\right)\right)=x_{1}, \ldots, q_{n}^{n}\left(Q_{n}\left(x_{1}, \ldots, x_{n}\right)\right)=x_{n}, \\
Q_{n}\left(q_{1}^{n}(x), \ldots, q_{n}^{n}(x)\right)=x .
\end{gathered}
$$

Отметим, что согласно следствиям 2,3 при $n \geqslant 3$ вьполняются включения: $Q_{n} \in \mathbf{C}_{\varphi}$, где $\varphi(t)=\lceil t / 2\rceil, q_{n}^{n} \in \mathbf{C}_{2 t}, q_{n-1}^{n} \in \mathbf{C}_{4 t}, \ldots, q_{2}^{n} \in \mathbf{C}_{2^{n-1} t}, q_{1}^{n} \in \mathbf{C}_{2^{n-1} t}$.

Используя свойства функций $Q_{n}$, нетрудно показать, что при любом $n, n \geqslant 2$, $\left[\mathbf{C}^{(n)}\right]=\mathbf{C}$. Более того, имеет место равенство $\left[\mathbf{C}^{(1)} \cup\left\{Q_{n}\right\}\right]=\mathbf{C}$. Ограничимся рассмотрением случая $n=2$.

Пусть $m \geqslant 2$ и $f\left(x_{1}, \ldots, x_{m}\right)$ - произвольная функция из $\mathbf{C}^{(m)}$. Положим $f_{1}(x)=$ $f\left(q_{1}^{m}(x), \ldots, q_{m}^{m}(x)\right)$. Тогда $f_{1} \in \mathbf{C}^{(1)}$ и в силу (3) имеет место тождество

$$
f_{1}\left(Q_{m}\left(x_{1}, \ldots, x_{m}\right)\right)=f\left(x_{1}, \ldots, x_{m}\right) .
$$

Таким образом, $f \in\left[\mathbf{C}^{(1)} \cup\left\{Q_{m}\right\}\right]$. Вместе с тем, как вытекает из определения, $Q_{m} \in$ $\left[\left\{Q_{2}\right\}\right]$. Следовательно, $f \in\left[\mathbf{C}^{(1)} \cup\left\{Q_{2}\right\}\right]$.

В связи с данным результатом возникает вопрос: для каких функций $\varphi$ класс $\mathbf{C}_{\varphi}^{(n+1)}$ будет целиком содержаться в классе $\left[\mathbf{C}_{\varphi}^{(n)}\right]$ ?

Теоремы 1-3 в известном смысле дают ответ на этот вопрос.

ТЕорема 1. Пусть $\alpha$ - положительное действительное число, $\varphi(t)$ - монотонно возрастающая функиия $u \varphi(t) \geqslant(1+\alpha) t$. Тогда для любых натуральных $m$, $n$ имеет место включение $\mathbf{C}_{\varphi(m)}^{(n+1)} \subset\left[\mathbf{C}_{\varphi}^{(2)}\right]$.

ДокАЗАТЕЛЬСТво. Зафиксируем $m, n$ и рассмотрим произвольную функцию $f\left(x_{1}, \ldots, x_{n+1}\right)$ из класса $\mathbf{C}_{\varphi}^{(n+1)}$. Положим

$$
f_{1}(x)=f\left(q_{1}^{n+1}(x), \ldots, q_{n+1}^{n+1}(x)\right) .
$$

Тогда

$$
f\left(x_{1}, \ldots, x_{n+1}\right)=f_{1}\left(Q_{n+1}\left(x_{1}, \ldots, x_{n+1}\right)\right) .
$$

Так как $Q_{2} \in \mathbf{C}_{\psi}$, где $\psi(t)=\lceil t / 2\rceil, Q_{n+1} \in\left[\left\{Q_{2}\right\}\right]$ и $\varphi(t) \geqslant(1+\alpha) t$, то $Q_{n+1} \in\left[\mathbf{C}_{\varphi}^{(2)}\right]$. Далее, $\left\{q_{1}^{n+1}, \ldots, q_{n+1}^{n+1}\right\} \subset \mathbf{C}_{2^{n} t}$. Значит, из леммы 1 выводим, что $f_{1} \in \mathbf{C}_{\tau}$, где $\tau(t) \stackrel{=}{=}$ $2^{n} \varphi^{(m)}(t)$. Поскольку $\varphi(t) \geqslant(1+\alpha) t$, найдется такоенатуральное $k$, что $\varphi^{(k)}(t) \geqslant \tau(t)$. Таким образом, для доказательства теоремы достаточно установить, что классу $\left[\mathbf{C}_{\varphi}^{(2)}\right]$ принадлежит любая функция из $\mathbf{C}_{\varphi^{(k)}}^{(1)}$.

Пусть $y=g(x)$ - произвольная функция из $\mathbf{C}_{\varphi(k)}^{(1)}$. Для любого $t, t \geqslant 1$, обозначим через $G_{t}$ такую булеву функцию,что

$$
y(t)=G_{t}\left(x(1), \ldots, x\left(\varphi^{(k)}(t)\right)\right) .
$$

Выберем теперь в классе $\mathbf{C}_{\varphi}$ функцию $z=h(x)$ таким образом, чтобы при любом $t$, $t \geqslant 1$, вьполнялось равенство $z(t)=x(\varphi(t))$. Если положить $v(t)=h^{(k)}(t)$, то при 
любом $t, t \geqslant 1$, будем иметь $v(t)=x\left(\varphi^{(k)}(t)\right)$. В классе $\mathbf{C}_{t}$ определим функцию $w=$ $g_{1}(x)$ условиями:

$$
w(s)= \begin{cases}G_{t}(x(1), \ldots, x(s)), & \text { если } s=\varphi^{(k)}(t), \\ 0 & \text { в противном случае. }\end{cases}
$$

Так как функция $\varphi$ строго монотонна, то определение функции $g_{1}$ корректно. Имеем теперь $g(x)=h^{(k)}\left(g_{1}(x)\right)$. Теорема доказана.

СлЕДСтвИЕ. В условиях теоремы 1 имеет место равенство

$$
\left[\mathbf{C}_{\varphi}^{(2)}\right]=\bigcup_{m, n \geqslant 1} \mathbf{C}_{\varphi^{(m)}}^{(n)}
$$

ДоКАЗАТЕЛЬСТво. Достаточно в одну сторону воспользоваться леммой 1 , а в другую - теоремой 1.

Пусть $f\left(x_{1}, \ldots, x_{n}\right) \in \mathbf{C}_{\varphi}$. Как отмечалось выше, для любого $t, t \geqslant 1$, существует такая булева функция $G_{t}$ от $n \varphi(t)$ переменных, что вьполняется равенство (1). Набор булевых функций $\left(G_{1}, \ldots, G_{t}\right)$, представляющий собой булевский $(n \varphi(t), t)$-оператор с $n \varphi(t)$ входными переменными и $t$ выходными переменными, будем называть $(\varphi(t), t)$-приближсением функции $f$. Как вытекает из определения, $(\varphi(t), t)$-приближение функции $y=f\left(x_{1}, \ldots, x_{n}\right)$ позволяет определить значения $y(1), \ldots, y(t)$ через значения $x_{i}(1), \ldots, x_{i}(\varphi(t)) \quad(1 \leqslant i \leqslant n)$ согласно равенствам (1). Поскольку число различных булевых функций $G_{t}$ равно $2^{2^{n \varphi(t)}}$, число возможных $(\varphi(t), t)$-приближений для функций из класса $\mathbf{C}_{\varphi}^{(n)}$ есть

$$
2^{2^{n \varphi(1)}} \cdot 2^{2^{n \varphi(2)}} \cdot \ldots \cdot 2^{2^{n \varphi(t)}}=2^{\sum_{i=1}^{t} 2^{n \varphi(i)}} .
$$

Если $M \subseteq \mathbf{C}_{\varphi}$, то через $l_{\varphi}(M, n, t)$ обозначим число различных $(\varphi(t), t)$-приближений для всех функций из множества $M^{(n)}$. Имеем, в частности,

$$
l_{\varphi}\left(\mathbf{C}_{\varphi}^{(n)}, n, t\right)=2^{\sum_{i=1}^{t} 2^{n \varphi(i)}} .
$$

Положим $\mathbf{C}_{\varphi}^{m, n}=\left(\mathbf{C}_{\varphi}^{(n)}\right)^{[m]}$.

Лемма 2. Пусть $\varphi(t) \leqslant t$. Для любых натуральных $m, n$ найдется такая положительнная константа d, что для всех натуральных р выполняется неравенство

$$
l_{\varphi}\left(\mathbf{C}_{\varphi}^{m, n}, p, t\right) \leqslant d \cdot\left(l_{\varphi}\left(\mathbf{C}_{\varphi}^{(n)}, n, t\right)\right)^{m}
$$

ДоКАЗАТЕЛЬСТво. Согласно следствию 2 все функции из $\mathbf{C}_{\varphi}^{m, n}$ входят в множество $\mathbf{C}_{\varphi}$. Поэтому для любой функции из $\mathbf{C}_{\varphi}^{m, n}$ понятие $(\varphi(t), t)$-приближения имеет смысл. Пусть $f \in \mathbf{C}_{\varphi}^{m, n}$. Предположим, что функция $f$ реализуется формулой $F$ над множеством $\mathbf{C}_{\varphi}^{(n)}$. Так как $\varphi(t) \leqslant t$, то для получения $(\varphi(t), t)$-приближения функции $f$ достаточно взять $(\varphi(t), t)$-приближения всех входящих в формулу $F$ функций и согласно строению формулы $F$ осуществить суперпозицию соответствующих булевских $(n \varphi(t), t)$-операторов. (При этом часть входных и выходных переменных у $(n \varphi(t), t)$-операторов может оказаться невостребованной, поскольку $\varphi(t)$ может быть строго меньше $t$.) Таким образом, для фиксированной формулы $F$ и для произвольных $n$-местных 
функций из $\mathbf{C}_{\varphi}^{(n)}$, входящих в эту формулу, число различных $(\varphi(t), t)$-приближений функций $f$, определяемых формулой $F$, не превосходит величины $\left(l_{\varphi}\left(\mathbf{C}_{\varphi}^{(n)}, n, t\right)\right)^{m}$. Если теперь обозначить через $d$ число различных формул $F$, содержащих $m$ символов $n$-местных функций (нам важна лишш структура формул $F$, поэтому при подсчете числа формул можно ограничиться использованием конкретных символов $n$-местных функций $f_{1}, \ldots$, $f_{m}$ и конкретных символов переменных $\left.x_{1}, \ldots, x_{m n-m+1}\right)$, то мы придем к неравенству (4). Лемма доказана.

Теорема 2. Пусть $\varphi(t) \leqslant t$ и функиия $\varphi$ неограничена. Тогда для любого $n$, $n \geqslant 1$, в классе $\mathbf{C}_{\varphi}^{(n+1)}$ найдется функиия, которую невозмохнно представить в виде суперпозиции функиий из класса $\mathbf{C}_{\varphi}^{(n)}$.

ДокАЗАТЕЛьСТво. Зафиксируем натуральное $n$ и определим функцию $y=f\left(x_{1}, \ldots\right.$, $\left.x_{n+1}\right)$ из класса $\mathbf{C}_{\varphi}^{(n+1)}$, которая не принадлежит классу $\left[\mathbf{C}_{\varphi}^{(n)}\right]$. Построение функции $f$ проведем по шагам, определяя на каждом шаге конечное число значений $y(t)$ с помощью подходящих булевых функций $G_{t}$ от переменных $x_{1}(1), \ldots, x_{n+1}(1), \ldots, x_{1}(\varphi(t))$, $\ldots, x_{n+1}(\varphi(t))$.

Пусть $N$ - множество натуральных чисел, $c(x, y)$ - какая-либо функция, осуществляющая взаимно-однозначное отображение $N^{2}$ на $N$.

ШаГ 1. Полагаем $t_{1}=1, y(1)=G_{1}\left(x_{1}(1), \ldots, x_{n+1}(1), \ldots, x_{1}(\varphi(1)), \ldots\right.$, $\left.x_{n+1}(\varphi(1))\right)=0$. Переходим к шагу 2 .

ШАГ $s+1$. Пусть $s=c(d, m)$ и уже определены число $t_{s}$ и величины $y(1), \ldots, y\left(t_{s}\right)$ с помошью булевых функций $G_{1}, \ldots, G_{t_{s}}$ :

$$
\begin{gathered}
y(1)=G_{1}\left(x_{1}(1), \ldots, x_{n+1}(1), \ldots, x_{1}(\varphi(1)), \ldots, x_{n+1}(\varphi(1))\right. \\
\ldots \ldots \ldots \ldots \ldots \ldots \ldots \ldots \ldots \ldots \ldots \ldots \ldots \ldots \ldots \ldots \ldots \ldots \\
y\left(t_{s}\right)=G_{t_{s}}\left(x_{1}(1), \ldots, x_{n+1}(1), \ldots, x_{1}\left(\varphi\left(t_{s}\right)\right), \ldots, x_{n+1}\left(\varphi\left(t_{s}\right)\right)\right) .
\end{gathered}
$$

Пользуясь тем, что функция $\varphi(t)$ монотонно не убывает и неограничена, выберем число $t_{s+1}$ столь большим, чтобы выполнялись неравенства $t_{s+1}>t_{s}$ и

$$
\log _{2} d+m \sum_{i=1}^{t_{s+1}} 2^{n \varphi(i)}<\sum_{i=t_{s}+1}^{t_{s+1}} 2^{(n+1) \varphi(i)}
$$

В правой части неравенства (5) стоит величина, равная логарифму (по основанию 2) числа всех булевских $\left((n+1) \varphi\left(t_{s+1}\right), t_{s+1}-t_{s}\right)$-операторов, $i$-й выход которых зависит от первых $(n+1) \varphi(i)$ входов $\left(t_{s}+1 \leqslant i \leqslant t_{s+1}\right)$. Поэтому если величина из левой части неравенства $(5)$ не меньше логарифма числа $\left(\varphi\left(t_{s+1}\right), t_{s+1}\right)$-приближений всех $(n+1)$-местных функций из класса $\mathbf{C}_{\varphi}^{m, n}$ (т.е. не меньше $\left.\log _{2}\left(l_{\varphi}\left(\mathbf{C}_{\varphi}^{m, n}, n+1, t_{s+1}\right)\right)\right)$, то можно найти такие булевы функции $G_{t_{s}+1}, \ldots, G_{t_{s+1}}$, зависящие соответственно от $(n+1) \varphi\left(t_{s}+1\right), \ldots,(n+1) \varphi\left(t_{s+1}\right)$ переменных, что $\left(\varphi\left(t_{s+1}\right), t_{s+1}\right)$-приближение функции $f\left(x_{1}, \ldots, x_{n+1}\right)$, определяемое через булевы функции $G_{1}, \ldots, G_{t_{s}}, G_{t_{s}+1}, \ldots, G_{t_{s+1}}$, будет отличаться от $\left(\varphi\left(t_{s+1}\right), t_{s+1}\right)$-приближений всех $(n+1)$-местных функций из $\mathbf{C}_{\varphi}^{m, n}$. В связи с этим определяем: если

$$
\log _{2}\left(l_{\varphi}\left(\mathbf{C}_{\varphi}^{m, n}, n+1, t_{s+1}\right)\right) \leqslant \log _{2} d+m \sum_{i=1}^{t_{s+1}} 2^{n \varphi(i)},
$$


то

$$
\begin{aligned}
y\left(t_{s}+1\right) & =G_{t_{s}+1}\left(x_{1}(1), \ldots, x_{n+1}(1), \ldots, x_{1}\left(\varphi\left(t_{s}+1\right)\right), \ldots, x_{n+1}\left(\varphi\left(t_{s}+1\right)\right)\right), \\
& \ldots \ldots \ldots \ldots \ldots \ldots \ldots \ldots \ldots \ldots \ldots \ldots \ldots \ldots \ldots \ldots \ldots \ldots \ldots \ldots \ldots \ldots \ldots \\
y\left(t_{s+1}\right) & =G_{t_{s+1}}\left(x_{1}(1), \ldots, x_{n+1}(1), \ldots, x_{1}\left(\varphi\left(t_{s+1}\right)\right), \ldots, x_{n+1}\left(\varphi\left(t_{s+1}\right)\right)\right),
\end{aligned}
$$

где $G_{t_{s}+1}, \ldots, G_{t_{s+1}}$ - выбранные указанньм вьше образом булевы функции. Если же неравенство (6) не выполняется, то полагаем

$$
\begin{aligned}
y\left(t_{s}+1\right) & =G_{t_{s}+1}\left(x_{1}(1), \ldots, x_{n+1}\left(\varphi\left(t_{s}+1\right)\right)\right)=0 \\
& \ldots \ldots \ldots \ldots \ldots \ldots \ldots \ldots \ldots \\
y\left(t_{s+1}\right) & =G_{t_{s+1}}\left(x_{1}(1), \ldots, x_{n+1}\left(\varphi\left(t_{s+1}\right)\right)\right)=0
\end{aligned}
$$

Переходим к шагу $s+2$.

По лемме 2 для всякого натурального $m$ (напомним, что число $n$ фиксировано) найдется такое $d$, что для любых натуральных $p$ выполняется неравенство (4). Так как левая часть неравенства (5) представляет собой логарифм (при $\left.t=t_{s+1}\right)$ правой части неравенства (4), то согласно построению на шаге $s+1$, где $s=c(d, m)$, функция $f\left(x_{1}, \ldots, x_{n+1}\right)$ будет определена таким образом, что она станет отличной от любой $(n+1)$-местной функции из класса $\mathbf{C}_{\varphi}^{m, n}$. Теорема доказана.

ЛЕмМА 3. Пусть $\varphi(t) \geqslant t u$ для любых натурального $k$ u положсительного действительного $\alpha$ найдется такое $t_{0}$, что при $t \geqslant t_{0}$ выполняется неравенство $\varphi^{(k)} \leqslant(1+\alpha) t$. Тогда для любых натуральных $m, n$ и полохительного действительного $\beta$ найдутся такие натуральные $t_{1} u$, что при $t \geqslant t_{1} u$ для всех натуральных р справедливо неравенство

$$
l_{\varphi^{(m)}}\left(\mathbf{C}_{\varphi}^{m, n}, p, t\right) \leqslant 2^{e \cdot 2^{n(1+\beta) t}} .
$$

ДокАЗАТЕЛЬСТво. Зафиксируем $m, n$ и рассмотрим произвольную функцию $y=$ $f\left(x_{1}, \ldots, x_{p}\right)$ из класса $\mathbf{C}_{\varphi}^{m, n}$. Из леммы 1 и неравенства $\varphi(t) \geqslant t$ следует, что $f \in$ $\mathbf{C}_{\varphi(m)}$. Пусть функция $f$ реализуется формулой $F$ над множеством $\mathbf{C}_{\varphi}^{(n)}$, содержащей $m$ символов $n$-местных функций:

$$
f\left(x_{1}, \ldots, x_{p}\right)=f_{0}\left(F_{1}, \ldots, F_{n}\right),
$$

где $f_{0} \in \mathbf{C}_{\varphi}^{(n)}$, а $F_{1}, \ldots, F_{n}$ - либо формулы над $\mathbf{C}_{\varphi}^{(n)}$, либо символы переменных $x_{1}, \ldots$, $x_{p}$. Предположим для определенности, что $F_{1}, \ldots, F_{r}$ - формулы, которые реализуют функции $y_{1}=f_{1}, \ldots, y_{r}=f_{r}$, a $F_{r+1}=x_{j_{1}}, \ldots, F_{n}=x_{j_{n-r}}$. По определению класса $\mathbf{C}_{\varphi}$ при любом $t, t \geqslant 1$, значения $y(1), \ldots, y(t)$ полностью определяются значениями

$$
\begin{gathered}
y_{1}(1), \ldots, y_{r}(1), x_{j_{1}}(1), \ldots, x_{j_{n-r}}(1), \ldots, y_{1}(\varphi(t)), \ldots, y_{r}(\varphi(t)) \\
x_{j_{1}}(\varphi(t)), \ldots, x_{j_{n-r}}(\varphi(t))
\end{gathered}
$$

Таким образом, для вычисления значений $y(1), \ldots, y(t)$ требуется, вообще говоря, иметь $(\varphi(t), t)$-приближение функции $f_{0}$. 
Переходя ко второму “ярусу" формулы $F$, предположим, что формула $F_{i}(1 \leqslant i \leqslant r)$ имеет вид

$$
F_{i}=g_{i}\left(G_{1}^{i}, \ldots, G_{n}^{i}\right),
$$

где $g_{i} \in \mathbf{C}_{\varphi}^{(n)}$, a $G_{1}^{i}, \ldots, G_{n}^{i}$ - либо формулы над $\mathbf{C}_{\varphi}^{(n)}$, либо символы переменных $x_{1}, \ldots$, $x_{p}$. Так как $\varphi(t) \geqslant t$, в данном случае для вычисления значений $y_{i}(1), \ldots, y_{i}(\varphi(t))$ необходимы, вообще говоря, значения $z_{s}(1), \ldots, z_{s}\left(\varphi^{(2)}(t)\right)$, если $G_{s}^{i}$ есть формула, реализующая функцию $z_{s}=h_{s}$, и значения $x_{j}(1), \ldots, x_{j}\left(\varphi^{(2)}(t)\right)$, если $G_{s}^{i}$ совпадает с переменной $x_{j}$. Таким образом, на этом этапе необходимо, вообще говоря, иметь $\left(\varphi^{(2)}(t), \varphi(t)\right)$ приближения функций $f_{i}(1 \leqslant i \leqslant r)$.

Поднимаясь далее по формуле $F$ “снизу вверх" и принимая во внимание, что $\varphi(t) \geqslant t$ и формула $F$ содержит $m$ символов функций из $\mathbf{C}_{\varphi}^{(n)}$, приходим к заключению, что для получения $\left(\varphi^{(m)}(t), t\right)$-приближения функции $f$ достаточно располагать $\left(\varphi^{(m)}(t), \varphi^{(m-1)}(t)\right)$-приближениями всех входящих в формулу $F$ функций.

Число всех $\left(\varphi^{(m)}(t), \varphi^{(m-1)}(t)\right)$-приближений функций из $\mathbf{C}_{\varphi}^{(n)}$ равно

$$
2^{\sum_{i=1}^{\varphi^{(m-1)}(t)} 2^{n \varphi(i)}}
$$

Следовательно, при любом натуральном $p$

$$
l_{\varphi(m)}\left(\mathbf{C}_{\varphi}^{m, n}, p, t\right) \leqslant d \cdot 2^{m \sum_{i=1}^{\varphi^{(m-1)}(t)} 2^{n \varphi(i)}},
$$

где $d$ есть количество всех формул, содержащих $m$ символов $n$-местных функций.

Согласно условиям леммы для выбранного $m$ и для положительного действительного $\alpha$ найдется такое $t_{2}$, что при $t \geqslant t_{2}$ вьполняется неравенство $\varphi^{(m-1)}(t) \leqslant(1+\alpha) t$. Поэтому, полагая

$$
e\left(t_{2}\right)=\sum_{i=1}^{\varphi^{(m-1)}\left(t_{2}\right)} 2^{n \varphi(i)},
$$

при $t \geqslant t_{2}$ будем также иметь

$$
\begin{aligned}
\sum_{i=1}^{\varphi^{(m-1)}(t)} 2^{n \varphi(i)} & =e\left(t_{2}\right)+\sum_{i=\varphi}^{\varphi^{(m-1)}\left(t_{2}\right)+1} 2^{n \varphi(i)} \\
& \leqslant e\left(t_{2}\right)+\sum_{i=1}^{(1+\alpha) t} 2^{n(1+\alpha) i} \leqslant e\left(t_{2}\right)+2^{n(1+\alpha)(1+(1+\alpha) t)} .
\end{aligned}
$$

Из (7) и (8) следует, что

$$
\log _{2}\left(l_{\varphi(m)}\left(\mathbf{C}_{\varphi}^{m, n}, p, t\right)\right) \leqslant \log _{2} d+m \cdot\left(e\left(t_{2}\right)+2^{n(1+\alpha)(1+(1+\alpha) t)}\right) .
$$

Возьмем теперь положительное действительное $\beta$ и выберем такие натуральные $t_{1}, e$ и положительное действительное $\alpha$, что $t_{1} \geqslant t_{2}$ и при $t \geqslant t_{1}$ вьполняется неравенство

$$
\log _{2} d+m e\left(t_{2}\right)+m 2^{n(1+\alpha)(1+(1+\alpha) t)} \leqslant e 2^{n(1+\beta) t} .
$$

Из сопоставления неравенств (7), (9) и (10) вытекает утверждение леммы. 
ТЕОРемА 3. Пусть $\varphi(t) \geqslant t u$ для любьх натурального $k$ и положительного действительного а существует такое $t^{\prime}$, что при $t \geqslant t^{\prime}$ выполняется неравенство $\varphi^{(k)}(t) \leqslant(1+\alpha) t$. Тогда для любого $n, n \geqslant 1$, в классе $\mathbf{C}_{\varphi}^{(n+1)}$ найдется функиия, которую невозмохсно представить в виде суперпозичии функиий из класса $\mathbf{C}_{\varphi}^{(n)}$.

ДокАЗАТЕЛьство. Зафиксируем натуральное $n$ и определим в классе $\mathbf{C}_{\varphi}^{(n+1)}$ функцию $y=f\left(x_{1}, \ldots, x_{n+1}\right)$, которая не принадлежит классу $\left[\mathbf{C}_{\varphi}^{(n)}\right]$. Так же, как и в теореме 2 , построение функции $f$ проведем по шагам, определяя на каждом шаге конечное число значений $y(t)$ в виде булевых функций $G_{t}$ от переменных $x_{1}(1), \ldots, x_{n+1}(1), \ldots$, $x_{1}(t), \ldots, x_{n+1}(t)$.

ШАГ 1. Полагаем $t_{1}=1, y(1)=G_{1}\left(x_{1}(1), \ldots, x_{n+1}(1)\right)=0$. Переходим к шагу 2.

ШАГ $m+1$. Пусть уже определены число $t_{m}$ и $y(1), \ldots, y\left(t_{m}\right)$ с помощью булевых функций $G_{1}, \ldots, G_{t_{m}}$ :

$$
\begin{aligned}
y(1) & =G_{1}\left(x_{1}(1), \ldots, x_{n+1}(1)\right) \\
& \ldots \ldots \ldots \ldots \ldots \ldots \ldots \ldots \\
y\left(t_{m}\right) & =G_{t_{m}}\left(x_{1}(1), \ldots, x_{n+1}(1), \ldots, x_{1}\left(t_{m}\right), \ldots, x_{n+1}\left(t_{m}\right)\right) .
\end{aligned}
$$

Согласно лемме 3 для данных $m, n$ найдутся такие натуральные $t_{0}$ и $e$, что при $t \geqslant t_{0}$ выполняется неравенство

$$
l_{\varphi(m)}\left(\mathbf{C}_{\varphi}^{m, n}, n+1, t\right) \leqslant 2^{e 2^{n(1+1 / n) t}} .
$$

Можно считать, что $t_{0} \geqslant t_{m}$. Выберем число $t_{m+1}$ так, чтобы выполнялись неравенства $t_{m+1}>t_{0}$ и

$$
e 2^{n(1+1 / n) t_{m+1}}<\sum_{i=t_{m}+1}^{t_{m+1}} 2^{(n+1) i}
$$

Поскольку число всех наборов $\left(G_{t_{m}+1}, \ldots, G_{t_{m+1}}\right)$ булевых функций, в которых функция $G_{i}\left(t_{m}+1 \leqslant i \leqslant t_{m+1}\right)$ зависит от $(n+1) i$ переменных, равно

$$
2^{\sum_{i=t_{m}+1}^{t_{m+1}} 2^{(n+1) i}}
$$

на основании неравенств (11) и (12) заключаем, что можно выбрать такие булевы функции $G_{t_{m}+1}, \ldots, G_{t_{m+1}}$ соответственно от $(n+1)\left(t_{m}+1\right), \ldots,(n+1) t_{m+1}$ переменных, что $\left(t_{m+1}, t_{m+1}\right)$-приближение функции $f\left(x_{1}, \ldots, x_{n+1}\right)$, определяемое через булевы функции $G_{1}, \ldots, G_{t_{m+1}}$, будет отличаться от всех $\left(\varphi^{(m)}\left(t_{m+1}\right), t_{m+1}\right)$-приближений функций из $\mathbf{C}_{\varphi}^{m, n}$ (в силу неравенства $\varphi(t) \geqslant t$ функции $G_{t_{m}+1}, \ldots, G_{t_{m+1}}$ можно считать зависяшими соответственно от $(n+1) \varphi^{(m)}\left(t_{m}+1\right), \ldots,(n+1) \varphi^{(m)}\left(t_{m+1}\right)$ переменньх). Полагаем

$$
\begin{aligned}
y\left(t_{m}+1\right) & =G_{t_{m}+1}\left(x_{1}(1), \ldots, x_{n+1}(1), \ldots, x_{1}\left(t_{m}+1\right), \ldots, x_{n+1}\left(t_{m}+1\right)\right) \\
& \ldots \ldots \ldots \ldots \ldots \ldots \ldots \ldots \ldots \ldots \ldots \ldots \ldots \ldots \ldots \ldots \ldots \ldots \ldots \ldots \ldots \\
y\left(t_{m+1}\right) & =G_{t_{m+1}}\left(x_{1}(1), \ldots, x_{n+1}(1), \ldots, x_{1}\left(t_{m+1}\right), \ldots, x_{n+1}\left(t_{m+1}\right)\right) .
\end{aligned}
$$

Переходим к шагу $m+2$. 
Непосредственно из построения видно, что для любого натурального $m$ на шаге $m+1$ функция $f\left(x_{1}, \ldots, x_{n+1}\right)$ определяется таким образом, что ее $\left(\varphi^{(m)}\left(t_{m+1}\right), t_{m+1}\right)$-приближение отличается от $\left(\varphi^{(m)}\left(t_{m+1}\right), t_{m+1}\right)$-приближения любой $(n+1)$-местной функции из $\mathbf{C}_{\varphi}^{m, n}$. Теорема доказана.

Из полученных результатов вытекает, что совокупность функций вида $(1+\alpha) t$ определяет своеобразную границу. Для функций $\varphi(t)$, расположенных ниже этой границы, включение $\mathbf{C}_{\varphi}^{(n+1)} \subset\left[\mathbf{C}_{\varphi}^{(n)}\right]$ невозможно ни при каком $n, n \geqslant 1$. Напротив, для функций $\varphi(t)$, лежаших вьше этой границы, соотношение $\mathbf{C}_{\varphi}^{(n+1)} \subset\left[\mathbf{C}_{\varphi}^{(n)}\right]$ (и даже соотношение $\left.\mathbf{C}_{\varphi}^{(n+1)} \subset\left[\mathbf{C}_{\varphi}^{(2)}\right]\right)$ вьполняется.

\section{СПИСОК ЦИТИРОВАННОЙ ЛИТЕРАТУРЫ}

[1] Яблонский С. В. Введение в теорию функций $k$-значной логики // Дискретная математика и математические вопросы кибернетики. I. М.: Наука, 1974. С. 9-66.

[2] Колмогоров А. Н. О представлении непрерывных функций нескольких переменных суперпозициями непрерывных функций меньшего числа переменных. // Докл. АН СССР. 1956. T. 108. № 2. C. 179-182.

[3] Арнольд В.И. О функциях трех переменных // Докл. АН СССР. 1957. Т. 114. № 4. C. $679-681$.

[4] Колмогоров А.Н. О представлении непрерьвных функций нескольких переменных в виде суперпозиций непрерывных функций одного переменного и сложения // Докл. АН СССР. 1957. T. 114. № 5. C. 953-956.

[5] Витушкин А. Г. К тринадцатой проблеме Гильберта // Докл. АН СССР. 1954. Т. 95. № 4. C. 701-704.

[6] Витушкин А. Г. О многомерных вариациях. М.: Гостехиздат, 1955.

[7] Колмогоров А.Н. Оценки минимального числа элементов $\varepsilon$-сетей в различных функциональных классах и их применение к вопросу о представимости функций нескольких переменных суперпозициями функций меньшего числа переменных // УМН. 1955. Т. 10. №1(63). C. $192-195$.

[8] Витушкин А. Г., Хенкин Г. М. Линейные суперпозиции функций // УМН. 1967. Т. 22. № 1. C. $77-124$.

[9] Vitushkin A. G. On representation of functions by means of superpositions and related topics // L'Enseignement math. 1977. V. 23. № 3-4. P. 255-320.

[10] Марченков С. С. Об одном методе анализа суперпозиций непрерывных функций // Проблемы кибернетики. 1980. Т. 37. С. 5-17.

[11] Гашков С. Б. О сложности приближенной реализации функциональных компактов в некоторых пространствах и о существовании функций с заданной по порядку сложностью // Фундаментальная и прикладная математика. 1996. Т. 2. № 3. С. 675-774.

[12] Яблонский С. В. Введение в дискретную математику. М.: Наука, 1986. 